\title{
Avaliação do Consumo Alimentar, Estado Nutricional e Ocorrência de Enteroparasitos em Crianças Pré-Escolares no Município de Picos-Piauí, Nordeste Brasileiro
}

\section{Assessment of Food Consumption, Nutritional Status and Enteroparasites Occurrence in Preschool Children in Picos-Piauí, Northeastern Brazil}

Ana Elisa Ramos; ; Tércia Tâmara Ribeiro da Silvaa; Ykaro Richard Oliveira*b; Paulo Henrique da Silvaa ; Maria Carolina de Abreu ; Marcia Maria Mendes Marquesa; Edson Lourenço da Silva ${ }^{c}$; Tamaris Gimenez Pinheiro ${ }^{a}$; Artemízia

Francisca de Sousa ${ }^{\mathrm{a}}$; Ana Carolina Landim Pacheco ${ }^{\mathrm{a}}$

aUniversidade Federal do Piauí. PI, Brasil.

'Instituto Federal de Educação, Ciência e Tecnologia do Piauí. PI, Brasil.

bUniversidade Estadual de Feira de Santana. PI, Brasil.

*E-mail: ykr-oliveira@hotmail.com

\begin{abstract}
Resumo
As parasitoses intestinais são sérios problemas de saúde pública, principalmente, em países em desenvolvimento. O objetivo do artigo foi verificar se a presença de enteroparasitoses influencia negativamente no estado nutricional das crianças da escola municipal N. Sra. Aparecida, na cidade de Picos-PI. Para isso, investigou-se a frequência de consumo alimentar, dados socioeconômicos, medidas antropométricas e material fecal de 28 crianças, com idade entre 3 e 6 anos. Os grupos de alimentos mais consumidos pelos envolvidos são: óleos, leite, carnes, leguminosas, cereais, bebidas e gorduras e de menor frequência: raízes e tubérculos, frutas, ovos, vegetais, açúcar e doces. Avaliando o estado nutricional se verificou que pelos parâmetros utilizados, Índice de Massa Corporal por idade (IMC/I), peso por idade (P/I) e altura por idade (A/I), a maioria se encontra normal. Os resultados do teste de fezes para parasitas intestinais mostraram que $92,85 \%$ das crianças apresentaram pelo menos um enteroparasita e apenas $7,15 \%$ não tinham contaminação, prevalecendo na maioria dos casos Ascaris lumbricoides. Portanto, estudos como este contribuem para o desenvolvimento de políticas públicas de prevenção e controle de doenças parasitárias que afetam o desenvolvimento infantil.
\end{abstract}

Palavras-chave: Avaliação Nutricional. Enteropatias. Saúde Escolar.

\begin{abstract}
Adequate childhood nutrition reflects in children's growth and physiological development, health and well-being. The aim of this study was to evaluate the habits and frequency of food intake, nutritional status and the occurrence of enteroparasites in preschool children from 3 to 6 years old from a public school in Picos, Piaui. Thus, the frequency of food intake, socioeconomic data, anthropometric measurements and fecal material of 28 children, aged 3 to 6 years were investigated. The most consumed food groups by those involved are: oils, milk, meat, pulses, cereals, beverages and fats and least consumed ones: roots and tubers, fruits, eggs, vegetables, sugar and sweets. Evaluating children's nutritional status through anthropometric indices, Body Mass Index for age (BMI/I), weight by age (P/I) and height by age (A/I), it was found that they were within the range of normality. Stool test results for intestinal parasites showed that $92.85 \%$ of children were positive for at least one enteroparasite and only $7.15 \%$ had no contamination, with the highest prevalence of Ascaris lumbricoides. Therefore, studies such as this contribute to the development of public policies for prevention and control of parasitic diseases that affect child's development.
\end{abstract}

Keywords: Nutritional Assessment. Intestinal Diseases. School Health.

\section{Introdução}

Uma alimentação adequada na infância se reflete no crescimento e no desenvolvimento fisiológico, na saúde e no bem-estar das crianças (LEAL et al., 2015). A fase pré-escolar é compreendida dos dois aos seis anos de idade, nesse período é frequente a inapetência, seletividade e indisciplina alimentar que podem levar a neofobia, além da redução da velocidade de crescimento estatural e redução de ganho ponderal. Essa fase é decisiva em termos de formação de hábitos alimentares que tendem a se solidificar na vida adulta, quando as escolhas alimentares são inapropriadas e podem acarretar carências nutricionais em curto e longo prazo (MELO; SILVA; SANTOS, 2018). A identificação do perfil de consumo alimentar, bem como do estado nutricional em escolares, constitui uma etapa fundamental para o estabelecimento de estratégias que visam reverter o atual quadro epidemiológico nutricional, visando a promoção da saúde e da alimentação saudável (MENÊSES et al., 2017).

O estado nutricional de crianças e jovens é comprometido pelas enteroparasitoses e, por consequência, o crescimento e o desenvolvimento das funções cognitivas dos indivíduos parasitados (LOPES et al., 2010). A população mais atingida e prejudicada pelas doenças parasitárias são as crianças, uma vez que, seus hábitos de higiene são, na maioria das vezes, inadequados e sua imunidade ainda não está totalmente eficiente para a eliminação dos parasitos (MENDES; SOUZA; MINÉ, 2017). As enteroparasitoses ainda constituem grave problema de Saúde Pública no Brasil, principalmente, pela sua correlação com o grau de desnutrição das populações, da falta de saneamento básico e água devidamente tratada (FERREIRA; ANDRADE, 2005; ROCHA et al., 2011; FONSECA et al., 2017). 
No estado do Piauí, alguns estudos demonstraram a prevalência de parasitos intestinais na população de São Raimundo Nonato (ALVES et al., 2003) e geronte de Parnaíba (FURTADO; MELO, 2011). Embora haja uma vasta literatura sobre a importância das enteroparasitoses para a Saúde Pública, e especialmente, em relação a escolares, pouca atenção tem sido dada ao assunto, nos programas de formação de educadores (FERREIRA; ANDRADE, 2005). Objetivou-se com este trabalho avaliar os hábitos de frequência do consumo alimentar, estado nutricional e a ocorrência de enteroparasitos em crianças pré-escolares de 3 a 6 anos de uma escola da rede pública de Picos, Piauí.

\section{Material e Métodos}

A pesquisa foi realizada entre os meses de agosto de 2011 e agosto de 2012, na Escola Municipal Nossa Senhora Aparecida, zona urbana do municipio de Picos-Piauí. A amostragem foi formada por 28 crianças do maternal, jardim I e II, com idade entre 3 e 6 anos de ambos os sexos. As crianças participaram mediante o consentimento dos seus respectivos responsáveis através da assinatura do Termo de Consentimento Livre e Esclarecido (TCLE). A pesquisa foi aprovada pelo Comitê de Ética em Pesquisa da Universidade Federal do Piauí, sob o parecer no 0036.0.045.000-11.

Para a avaliação do perfil socioeconômico, os responsáveis pelas crianças responderam a um questionário com dados socioeconômicos da família (renda familiar, grau de escolaridade, além de informações referentes à moradia, abastecimento de água, esgoto, coleta de lixo e hábitos de higiene). A frequência do consumo alimentar foi analisado da seguinte maneira: frequência $<4$ vezes por semana considerase como consumo não habitual $\mathrm{e} \geq 4$ vezes por semana como consumo habitual. O estado nutricional das crianças foi avaliado segundo os índices antropométricos peso/idade (P/I), altura/idade (A/I) e Indice de Massa Corporal para Idade (IMC/I), segundo os critérios adotados pela Organização Mundial da Saúde (WHO, 2002).

Os exames parasitológicos de fezes foram realizados no Laboratório de Parasitologia da Universidade Federal do Piauí, Campus Senador Helvídio Nunes de Barros, utilizando-se a técnica da Centrífugo-Sedimentação pelo Formaldeido-Éter (RITCHIE, 1948; ALLEN; RIDLEY, 1970). Os resultados foram analisados por meio de tabela de frequência de parasitos e da associação dos parasitos com o estado nutricional, verificados pelo teste exato de Fisher com margem de $90 \%$ de confiança, usando o software $\mathrm{R}$.

\section{Resultados e Discussão}

O estudo foi constituído por 28 crianças com idade de 3 a 6 anos. No que concerne à renda familiar mensal, observouse que $71,40 \%$ das famílias possuiam renda menor ou igual a um salário mínimo. Com relação ao nível de escolaridade dos responsáveis pelas crianças, verificou-se que 39,29\% possuiam Ensino Fundamental incompleto, seguido por 28,57\% com o Ensino Médio completo e 3,57\% não possui nenhum grau de escolaridade, ou seja, analfabetos. Nenhum dos participantes possuia Ensino Superior completo (Quadro $1)$.

Quadro 1 - Distribuição da população estudada segundo dados socioeconômicos.

\begin{tabular}{|c|l|c|}
\hline Variáveis & \multicolumn{1}{|c|}{ Respostas } & $\begin{array}{c}\text { Frequência } \\
(\%)\end{array}$ \\
\hline \multirow{4}{*}{$\begin{array}{c}\text { Renda } \\
\text { Familiar }\end{array}$} & Menos de 1 salário mínimo & 35,70 \\
\cline { 2 - 3 } & 1 salário mínimo & 35,70 \\
\cline { 2 - 3 } & 1 a 2 salários mínimos & 17,90 \\
\cline { 2 - 3 } & 2 a 5 salários mínimos & 10,70 \\
\cline { 2 - 3 } & Mais de 5 salários mínimos & 0,00 \\
\hline \multirow{4}{*}{$\begin{array}{c}\text { Escolaridade } \\
\text { dos Pais }\end{array}$} & Analfabeto & 3,57 \\
\cline { 2 - 3 } & Ensino Fundamental Incompleto & 39,29 \\
\cline { 2 - 3 } & Ensino Fundamental Completo & 10,71 \\
\cline { 2 - 3 } & Ensino Médio Incompleto & 14,29 \\
\cline { 2 - 3 } & Ensino Médio Completo & 28,57 \\
\cline { 2 - 3 } & Superior Incompleto & 3,57 \\
\cline { 2 - 3 } & Superior Completo & 0,00 \\
\hline
\end{tabular}

Fonte: Dados da pesquisa

A partir da investigação socioeconômica dos participantes, a população foi caracterizada com baixo poder aquisitivo, baixo nível de escolaridade e até analfabetos. Avaliou-se as condições de moradia das crianças e $100 \%$ das casas estão localizadas em um bairro no qual não há uma totalidade de serviço de água tratada e rede de esgoto. As condições de saneamento e moradia inadequada podem ser relacionadas às condições de saúde de uma população, uma vez que a ausência de tratamento sanitário adequado e condições de moradia precárias podem favorecer o aparecimento de doenças infecciosas, principalmente, as diarreias na infância, que podem influenciar de forma negativa o crescimento e desenvolvimento infantil (CARNEIRO; ALCANTARA, 2017). É relevante ressaltar que a capacidade dos pais em cuidar dos seus filhos está ligada ao acesso ao trabalho e melhores condições de moradia (FERREIRA et al., 2006).

Para Molina et al. (2010), as condições socioeconômicas das famílias determinam, em grande parte, o consumo e a qualidade da alimentação das crianças. Analisando a frequência (\%) de consumo alimentar, observou-se que os grupos de alimentos de consumo $\geq 4$ vezes por semana foram: leite e derivados; carnes e similares; leguminosas; cereais e derivados; bebidas; óleos e gorduras (Quadro 2). Estes alimentos são priorizados na compra de pessoas com baixa renda e são as principais fontes de macronutrientes, com exceção de bebidas como o refrigerante e suco artificial. Os grupos considerados de menor frequência foram: ovos; vegetais verdes e amarelos; outros vegetais; outras bebidas; outros derivados de cereais; açúcar, rapadura, caldo de cana; doces e similares e frutas (Quadro 2). Frutas e vegetais são fontes de micronutrientes e fibras, importantes no crescimento e desenvolvimento infantil. Segundo a Organização Mundial 
de Saúde (WHO, 2002), o baixo consumo de frutas e vegetais está entre os principais fatores que levam ao desenvolvimento de morbidades no mundo.

Quadro 2 - Consumo alimentar por relação de alimentos da população estudada

\begin{tabular}{|l|c|c|}
\hline Alimento/Bebida & $\begin{array}{c}<4 \text { vezes por } \\
\text { semana Freq. } \\
(\%)\end{array}$ & $\begin{array}{c}4 \text { vezes por } \\
\text { semana Freq. } \\
(\%)\end{array}$ \\
\hline Leites e derivados & 17,86 & 82,14 \\
\hline Carnes e similares & 17,86 & 82,14 \\
\hline Ovos & 75,00 & 25,00 \\
\hline Vegetais verdes e amarelos & 75,00 & 25,00 \\
\hline Outros vegetais & 67,86 & 32,14 \\
\hline Leguminosas & 28,57 & 71,43 \\
\hline Cereais e derivados & 39,29 & 60,71 \\
\hline $\begin{array}{l}\text { Outros derivados de } \\
\text { cereais (pizza, coxinha, } \\
\text { pastel, etc.) }\end{array}$ & 64,29 & 35,71 \\
\hline Bebidas & 39,29 & 60,71 \\
\hline $\begin{array}{l}\text { Outras bebidas (chás e } \\
\text { cafés) }\end{array}$ & 67,86 & 32,14 \\
\hline $\begin{array}{l}\text { Açúcar, rapadura e caldo } \\
\text { de cana }\end{array}$ & 57,14 & 42,86 \\
\hline Doces e similares & 53,57 & 46,43 \\
\hline Gorduras & 46,43 & 53,57 \\
\hline Óleos & 10,71 & 89,29 \\
\hline Frutas & 75,00 & 25,00 \\
\hline
\end{tabular}

Fonte: Dados da pesquisa

O guia alimentar da população brasileira abaliza o consumo de frutas, legumes e verduras como sendo o único fator protetor na alimentação. A infância é uma fase vital para mudanças nos hábitos alimentares e estilos de vida, os quais, por sua vez, poderão repercutir no futuro, em escolhas mais saudáveis (OSORIO et al., 2002).

A valiação do estado nutricional das crianças, segundo os parâmetros P/I, A/I e IMC/I estão apresentados no gráfico da Figura 1. Através do IMC foi registrado uma alta prevalência de adequação antropométrica $(67,80 \%)$, dados que corroboram com os obtidos por Costa et al. (2018), com crianças com idade entre 8 e 10 anos, de uma escola da rede privada de ensino do município de Picos-PI e Barros et al. (2016), que traçaram um perfil nutricional de alunos com idade de 4 a 19 anos de Escolas Públicas do município de Vera Mendes-PI, que também obtiveram prevalência dos padrões de eutrofia. Em 28,60\% das crianças foi comprovada a desnutrição (magreza e magreza acentuada) e em 3,5\% obesidade (Figura 1). A desnutrição está relacionada a mais de um terço de todas as mortes de crianças no mundo, embora raramente seja listada como a causa direta. Por outro lado, a obesidade é considerada como um dos quatro principais fatores de risco para as Doenças Crônicas não Transmissíveis (DCNT), sendo mais agravante quanto mais precoce a sua ocorrência.
Gráfico 1 - Classificação do estado nutricional das crianças seguindo os parâmetros antropométricos (OMS 2006/2007).

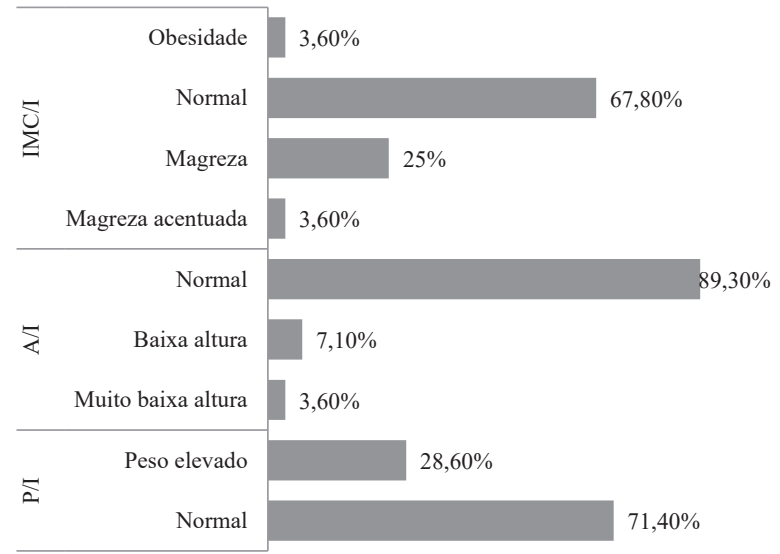

Fonte: Dados da pesquisa

Em razão disso, torna-se importante o estudo do estado nutricional de crianças, haja vista a comprovada associação das condições de nutrição na fase infantojuvenil com os níveis de saúde na idade adulta (PEREIRA et al., 2017).

Analisando o estado nutricional através do índice antropométrico peso por idade (P/I), as crianças pré-escolares estão em maioria dentro da normalidade $(71,70 \%)$ e uma parcela $(28,60 \%)$ com peso elevado. Em relação a altura para a idade $(\mathrm{A} / \mathrm{I})$, a maioria das crianças apresenta os índices de normalidade (89,30\%) (Figura 1). A avaliação do crescimento é a medida que melhor define a saúde e o estado nutricional de crianças, já que distúrbios na saúde e nutrição, independentemente de suas etiologias, invariavelmente afetam o crescimento infantil (SIGULEM; DEVINCENZI; LESSA, 2000).

Resultados dos exames de fezes para parasitas intestinais demonstraram que $92,85 \%$ das crianças pré-escolares (3 a 6 anos) de uma escola da rede pública de Picos-PI se apresentaram positivas para a presença de ao menos um enteroparasito e apenas 7,15\% não apresentam contaminação (Quadro 3).

Quadro 3 - Prevalência de enteroparasitas em crianças (3 a 6 anos) de uma escola da rede pública no municipio de Picos-PI.

\begin{tabular}{|l|c|}
\hline \multicolumn{1}{|c|}{ Parasita } & Frequência (\%) \\
\hline Ascaris lumbricoides & 71,4 \\
\hline Ancilostomídeos & 64,5 \\
\hline Fasciola hepática & 42,9 \\
\hline Strongyloides stercoralis & 25,0 \\
\hline Baladium coli & 21,4 \\
\hline Enterobius vermicularis & 14,2 \\
\hline Entamoeba histolytica/dispar & 10,7 \\
\hline Taenia ssp. & 10,7 \\
\hline Trichuris trichura & 3,5 \\
\hline
\end{tabular}

Fonte: Dados da pesquisa

OQuadro3demonstraasprincipais espécies diagnosticadas: Ascaris lumbricoides (71,4\%), Ancilostomídeos (64,5\%) e Fasciola hepática (42,9\%). Estudo realizado por Seixas et al. (2011) com escolares de uma área periurbana de Salvador- 
BA apresentou déficit do estado nutricional e a maioria se encontrava parasitada, prevalecendo a frequência de Ascaris lumbricoides, Trichuris trichiura, Enterobius vermicularis, Strongyloides stercoralis e Ancilostomídeos, helmintos que também foram encontrados nos escolares deste estudo. $\mathrm{O}$ Ascaris lumbricoides foi o parasita mais frequente neste estudo, o risco de um quadro de desnutrição é corrente aos pacientes portadores dessa enfermidade e principalmente em crianças, pois a persistência do parasita exige um elevado desvio de importantes nutrientes, como proteínas, vitaminas e carboidratos (SILVA et al., 2011).

Estudos revelam a prevalência de casos de enteroparasitoses em sujeitos subjugados à renda financeira e escolaridade mais baixa (CABRAL-MIRANDA et al., 2010; ARAÚJO FILHO et al., 2011; DIAS et al., 2017; MORI et al., 2016) acordando com os resultados apresentados neste estudo. A maioria das crianças era de famílias com baixo poder aquisitivo, baixo nível de escolaridade. Outros fatores como condições de moradia (falta de água tratada e rede de esgoto) e hábitos alimentares (lavar as mãos antes de se alimentar) precários evidenciam um alto grau de parasitismo nas crianças avaliadas. Condições de saneamento básico, principalmente aquelas referentes à coleta e ao armazenamento da água utilizada para consumo, ao destino do lixo caseiro e a hábitos de higiene, são fatores que não só favorecem a elevada prevalência das parasitoses intestinais, bem como contribuem para a manutenção dessas (VISSER et al., 2011).

Foi também avaliado o modo de lavagem de verduras, especialmente as folhosas que são consumidas pela família, em $91 \%$ dos casos os responsáveis pela criança lavava as verduras somente com água e apenas $9 \%$ higienizavam corretamente as verduras com solução de hipoclorito de sódio. Em relação aos hábitos de higiene pessoal das 28 crianças, apenas $10 \%$ destas lavavam as mãos antes de manipular qualquer tipo de alimento. As enteroparasitoses acarretam imensos prejuízos à saúde do indivíduo parasitado, principalmente, em crianças que não possuem hábitos higiênicos constantes e defesa imunológica ineficaz contra esses parasitos. O principal dano das parasitoses intestinais em crianças em idade escolar é o acometimento do desenvolvimento físico e mental, dificultando assim, o começo do aprendizado (MELO; FERRAZ; ALEIXO, 2010).

Este estudo foi o primeiro a fazer análise parasitológica em crianças do municipío de Picos com idade pré-escolar (3 a 6 anos) e constatou uma alta prevalência (92, 85\%) de crinças parasitadas. Estudo realizado anteriormente por Furtado e Melo (2011), em Parnaíba-PI, verificou também uma alta prevalência de enteroparasitoses agora em idosos, indicando um estado epidemiológico preocupante. Apesar da infecção por enteroparasitos ser adquirida em qualquer idade, constata-se que essa ocorre nos primeiros anos de vida. No Brasil, mais da metade de crianças pré-escolares e escolares se encontram parasitadas, podendo ser justificada também pela falta de acesso aos serviços básicos de saúde e pela ausência de projetos de educação sanitária (FERREIRA; ANDRADE, 2005; MASCARINO; DONALÍSIO, 2006; ANDRADE et al., 2010).

Sabe-se que vários fatores podem contribuir para o fracasso escolar das crianças, entre os quais podem ser citados: o estado nutricional das crianças, baixa renda familiar, escolaridade dos pais, condições higiênico-sanitárias, bem como a presença de parasitas intestinais que são responsáveis por limitar a capacidade pessoal dos sujeitos acometidos (MACHADO et al., 2013; ANTUNES; LIBARDONI, 2017).

\section{Conclusão}

Bons habitos alimentares devem ser desenvolvidos em crianças na idade pré-escolar e os resultados apotam a necessidade de intervenções na escola para promoção da saúde das crianças. O estado nutricional das crianças se apresentava dentro da faixa de normalidade, confirmando um estado de transição nutricional de desnutridos para eutrofia em uma região do Nordeste brasileiro. A grande maioria das crianças estava infectada com algum tipo de enteroparasito, tendo a maior prevalencia de Ascaris lumbricoides. Os parasitos são os principais causas de morbidade infantil, acarretando diminuição no desenvolvimento físico e mental, comprometendo o aprendizado das crianças. Portanto, estudos como esse contribuem para o desenvolvimento de Políticas Públicas de prevenção e controle de doenças parasitárias que afetam o desenvolvimento infantil.

\section{Referências}

ALLEN, A.V.H.; RIDLEY, D.S. Further observations on the fortiol-ether concentration technique for faccal parasites. J. Clin. Pathol., v.23, p.545-546, 1970. doi: 10.1136/jcp.23.6.545

ALVES, J.R. et al. Parasitoses intestinais em região semi-árida do Nordeste do Brasil: resultados preliminares distintos das prevalências esperadas. Cad. Saúde Pública, v.19, n.2, p.667670, 2003.

ANDRADE, E.C. et al. Parasitoses intestinais: uma revisão sobre seus aspectos sociais, epidemiológicos, clínicos e terapêuticos. Rev. APS, v.13, n.2, p.231-240, 2010

ANTUNES, A.S.; LIBARDONI, K.S.D.B. Prevalence of enteroparasitosis in children of day-care center in the municipality of Santo Angelo, RS. Rev. Contexto Saúde, v.17, n.32, p.144-156, 2017. doi: 10.21527/2176-7114.2017.32.144-156

ARAÚJO FILHO, H.B. et al. Parasitoses intestinais se associam a menores índices de peso e estatura em escolares de baixo estrato socioeconômico. Rev. Paul. Pediatr., v.29, n.4, p.521-528, 2011.

BARROS, K.C.S. et al. Perfil socioeconômico e nutricional de escolares em (in)segurança alimentar de uma região do Semiárido Nordestino. Rev. Bras. Ciênc. Saúde, v.20, n.1, p.5-10, 2016. doi: 10.4034/RBCS.2016.20.01.01

CABRAL-MIRANDA, G.; DATTOLI, V.C.C.; DIAS-LIMA, A. Enteroparasitos e condições socioeconômicas e sanitárias em uma comunidade quilombola do semiárido baiano. Rev. Patol. Trop., v.39, n.1, p.48-55, 2010

CARNEIRO, A.I.O.; ALCANTARA, J.R. Perfil socioeconômico e antropométrico de crianças de famílias assistidas pelo bolsa família matriculadas em creches municipais conveniadas da 
regional I de Fortaleza-CE. III Simpósio de Pesquisa em Ciências Médicas (UNIFOR), 2017.

COSTA, M.C. et al. Estado nutricional, práticas alimentares e conhecimentos em nutrição de escolares. Rev. Atenção Saúde, v.16, n.56, p.12-17, 2018. doi: 10.13037/ras.vol16n56.4811

DIAS, S.M. et al. Prevalência de parasitoses intestinais em crianças atendidas em uma unidade de saúde da família em Belém, Pará, Brasil. Rev. Multiprof. Saúde Hosp. São Marcos, v.2, n.1, p.17-25, 2017.

DIAS, S.M. et al. Prevalência de parasitoses intestinais em crianças atendidas em uma unidade de saúde da família em Belém, Pará, Brasil. Rev. Multiprof. Saúde Hospital São Marcos, v.2, n.1, p.17-25, 2017.

FERREIRA, G.R.; ANDRADE, C.F.S. Alguns aspectos socioeconômicos relacionados a parasitoses intestinais e avaliação de uma intervenção educativa em escolares de Estiva Gerbi, SP. Rev. Soc. Bras. Med. Trop., v.38, n.5, p.402-405, 2005. doi: 10.1590/S0037-86822005000500008.

FERREIRA, H. et al. Estudo epidemiológico localizado da freqüência e fatores de risco para enteroparasitoses e sua correlação com o estado nutricional de crianças em idade préescolar. Publicatio UEPG, v.12, n.4, p.33-40, 2006. doi: 10.5212/ Publ.Biologicas.v.12i4.0004

FONSECA, R.E.P.; BARBOSA, M.C.R; FERREIRA, B.R. High prevalence of enteroparasites in children from Ribeirão Preto, São Paulo, Brazil. Rev. Bras. Enferm., v.70, n.3, p.566-571, 2017. doi: 10.1590/0034-7167-2016-0059

FURTADO, L.F.V.; MELO, A.C.F.L. Prevalência e aspectos epidemiológicos de enteroparasitoses na população geronte de Parnaíba, Estado do Piauí. Rev. Soc. Bras. Med. Trop., v.44, n.4, p.513-515, 2011.

LEAL, K.K. et al. Qualidade da dieta de pré-escolares de 2 a 5 anos residentes na área urbana da cidade de Pelotas, RS. Rev Paul. Pediatr., v.33, n.3, p.310-317, 2015. doi: 10.1016/j. rpped.2015.05.002 0103-0582

LOPES, L.M. Ocorrência de parasitas e comensais intestinais em crianças da comunidade da Vila Inglesa, em São Paulo, SP, Brasil. Rev. Inst. Adolfo Lutz, v.69, n.2, p.252-254, 2010.

MACHADO, E.R.; CAMPOS, R.R.; NASCIMENTO, V.V. Enteroparasitoses entre escolares da cidade de Águas Lindas de Goiás. Ensaios Ciênc., v.17, n.5, p.19-32, 2013.

MASCARINO, L.M.; DONALÍSIO, R.M. Giardíase e criptosporidiose em crianças institucionalizadas em creches no Estado de São Paulo. Rev Soc. Bras. Med. Trop., v.39, n.6, p.577579, 2006. doi: 10.1590/S0037-86822006000600015.

MELO, E.M.; FERRAZ, F.N.; ALEIXO, D.L. Importância do estudo da prevalência de parasitos intestinais de crianças em idade escolar. SaBios Rev. Saúde Biol.., v.5, n.1, p.43-47, 2010.

MELO, K.S.; SILVA, K.L.G.D.; SANTOS, M.M.D. Avaliação do estado nutricional e consumo alimentar de pré-escolares e escolares residentes em Caetés-PE. Rev. Bras. Obesidade, Nutr. Emagrec., v.12, n.76. Supl. 2. p.1039-1049, 2018.
MENDES, G.R.; SOUZA, J.A. MINÉ, J.C. Diagnóstico de parasitos patogênicos e não patogênicos em crianças do município de Ponta Grossa- PR (2012-2016). Anais do 15 CONEX. 2017.

MENÊSES, L.E.N. et al. Consumo alimentar e estado nutricional de crianças em uma escola privada de Palmas, Tocantins. Rev. Desafios, v.4, n.3, p.43-51, 2017. doi: 10.20873/uft.23593652.2017v4n3p43

MOLINA, M.C.B. et al. Preditores socioeconômicos da qualidade da alimentação de crianças. Rev. Saúde Pública, v.44, n.5, p.78592, 2010. doi: 10.1590/S0034-89102010005000036

MORI, F.M.R.L. et al. Fatores associados a enteroparasitoses em escolares da rede municipal de ensino de Cambé. Semina Ciênc. Biol. Saúde, v.37, n.1, p.15-24, 2016. doi: 10.5433/1679-0367.2016v37n1p15

OSORIO, E.J.; WEISSTAUB, N.G.; CASTILLO, D.C. Desarrollo de conducta alimentaria en la infancia y sus alteraciones. Rev. Chilena Nutr., v.29, n.3, p.280-85, 2002. doi: 10.4067/S071775182002000300002

PEREIRA, I.F.S. et al. Estado nutricional de menores de 5 anos de idade no Brasil: evidências da polarização epidemiológica nutricional. Ciênc. Saúde Coletiva, v.22, n.10, p.3341-3352, 2017. doi: 10.1590/1413-812320172210.25242016

RITCHIE, L.S. An ether sedimentation technique for routine stool examination. Bull. US Army Med. Depart., v.8, n.4, p.326, 1948.

ROCHA, T.J.M. et al. Relação entre aspectos socioeconômicos e a ocorrência de ectoparasitoses e enteroparasitoses em uma comunidade do litoral norte alagoano. Rev. Bras. Anal. Clín., v.43, n.4, p.271-276, 2011.

SEIXAS, M.T.L. et al. Avaliação da frequência de parasitos intestinais e do estado nutricional em escolares de uma área periurbana de salvador, Bahia, Brasil. Rev. Patol. Trop., v.40, n.4, p.304-314, 2011. doi: 10.5216/rpt.v40i4.16762

SIGULEM, D.M.; DEVINCENZI, M.U.; LESSA, A.C. Diagnóstico do estado nutricional da criança e do adolescente. $J$. Pediatr., v.76, Supl.3, p. 275-284, 2000.

SILVA, J.C. et al. Parasitismo por Ascaris lumbricoides e seus aspectos epidemiológicos em crianças do Estado do Maranhão. Rev. Soc. Bras. Med. Trop., v.44, n.1, p.100-102, 2011. doi: 10.1590/S0037-86822011000100022

SILVA, J.C. et al. Parasitismo por Ascaris lumbricoides e seus aspectos epidemiológicos em crianças do Estado do Maranhão. Rev. Soc. Bras. Med. Trop., v.44, n.1, p.100-102, 2011. doi: 10.1590/S0037-86822011000100022

VISSER, S. et al. Estudo da associação entre fatores socioambientais e prevalência de parasitose intestinal em área periférica da cidade de Manaus (AM, Brasil). Ciênc. Saúde Coletetiva, v.16, n.8, p.3481-3492, 2011. doi: 10.1590/S141381232011000900016

WORLD HEALTH ORGANIZATION. The world health report 2002: reducing risks, promoting healthy life. Geneva: WHO; 2002. 